\title{
Influence of resistance of contact units of switching devices on the electric power losses in shop networks of low tension
}

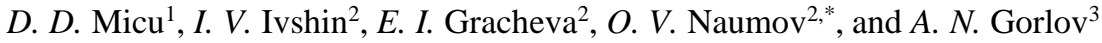 \\ ${ }^{1}$ Technical University of Cluj-Napoca, Cluj-Napoca, Romanian \\ ${ }^{2}$ Kazan State Power Engineering University, Kazan, Russia \\ ${ }^{3}$ The Southwest State University, Kursk, Russia
}

\begin{abstract}
This paper presents calculation of resistance of tightening contact joints of switching devices. It allows considering the technical condition of low-voltage switching equipment and to specify energy emitted in the switching device in the mode of electrical networks operation is presented in the article.
\end{abstract}

\section{Introduction}

Electric power distribution in shop networks between receivers and power source operation controls, transmission lines and receivers is carried out by means of electric devices. Electric devices do not perform direct working functions of units, but, nevertheless, they are extremely important and integral parts of this device which to a large extent drives the correct, exact and reliable work of an execution part of the device. Therefore, one can separate the electric device from the whole device, and represent it as independent unit only conditionally.

The concept of "electric contact" means the reliable connection of two conductors allowing current flow. Researchers have been always interested in the processes occurring on the surface of engagement when switching an electrical circuit [1].

The science dealing with electric contact is based on studying the mechanical, chemical, thermal and electric processes occurring on the adjoining surfaces. First of all, mechanical properties of contact materials induce the structure of surfaces and its influence on the area of engagement. Chemical properties are expressed by the fact that on the surface of contacts oxide, sulphidic or other protective films can be formed. Thermal and electrophysical processes on contacts are driven by a current flow and can be shown in the form of Joule-Lenz law, Thompson, Peltier's effects, etc. [2].

The purpose for creation of any electrical connection consists in forming the contact of two conductors at which electrons of a crystal lattice of one of them can freely pass into the lattice of the other one. One of the main characteristics of metal surface is its roughness. Metal surfaces always have roughnesses irrespective of a way of its processing. The nature of the contact of two solids largely depends on the roughness of the contacting surfaces, which, first of all, conjugate each other in those places where the microprotrusion of one surface meets the corresponding microprotrusion of the other. Intense local pressure will cause metal deformation in these microregions of contact points. During further surfaces approaching, the area of contact units will increase and create new contact spots during contacting by other roughnesses. All processes occur until forces of crushing resistance are not equal to the external applied force. But even when this process is finished and there is a considerable deformation of metal, nevertheless the major part of the adjoining surfaces is separated from each other by the distances many times exceeding distances on which interatomic forces begin to act. Only the small platform provides the real metal tip at the expense of the available microroughnesses [3].

\section{Calculation of the resistance of the contact groups of switching devices}

While disconnecting the contacts the surface area of contact begins to decrease, and the current density in the tightening area increases in the process of contacts discrepancy. The Joule heat which is selected in this area increases so quickly that microroughnesses manage to melt and form the liquid metal conducting bridge between contacts [4]. Under the influence of surface tension forces, the bridge first has a barrel-shaped shape, which, as the contacts diverge, turns into a hyperbolic with a saddle approximately in the middle of the bridge. Such bridge shapes were considered by Frank Llewellyn-Jones during slow formation of contacts. For a large number of metals Jones experimentally confirmed justice of the formula of Ragnar Holm received on the basis of Wiedemann-Franz-Lorenz law $[5,14]$ :

$$
\alpha\left(T_{\mathrm{p}}^{2}-T_{0}^{2}\right)=\frac{U^{2}}{4}
$$

\footnotetext{
* Corresponding author: 311670@list.ru
} 
where $T_{\mathrm{p}}$ is the bridge temperature before its destruction; $T_{0}$ is the temperature of the opposite end of contact; $U$ is the voltage drop on the bridge; $\alpha=2.4 \cdot 10^{-8} \mathrm{~V} /$ degree $^{2}$ is the value of the constant which received the name of L. Lorentz. Its value was specified by means of quantum statistics by A. Sommerfeld. This formula expresses relationship between temperature and voltage drop at any section of liquid threw, in this case on the melted bridge.

Some types of low-voltage switching devices, despite the seeming simplicity, in some cases represent very complex technical system, the main requirement to which is normal functioning according to specific assignment. The general provision defining functional suitability of the device assumes satisfaction of predefined and absolutely certain criteria requirements during its operation, and according to contents and rigidity they can strongly differ depending on type of the device, the modes and conditions of its operation [6].

For example, characteristic of time cut-outs is the possibility of long finding of their contacts in the closed status, and in the conditions of action of aggressive environments, moisture, increased temperature, etc. It, in turn, causes strict requirements to stability and level of transition resistance of contact joints.

For contactors these requirements can be softened as at frequent operational switching of current a periodic updating of contact surfaces becomes possible. But in both cases the availability of information on contact joint resistance is necessary because of large extension and branching of factory networks of low tension with a set of consecutive nodes with contact joints the share of resistance of the last in the general equivalent resistance of intra shop networks is rather high. Therefore, when determining electric power losses in factory networks up to $1000 \mathrm{~V}$ it is necessary to consider resistance of a contact system of switching devices. The power actually consumed by the device during its functioning and disseminated in it should be minimal. Low-voltage apparatus engineering has recently begun to develop quite intensively. There is a replacement of outdated switching devices by devices of new generation to high current limiting capacity, namely the devices providing higher reliability and profitability [7].

It is known that the electrical wear resistance and operability of the contacts of devices depend on many factors: the contacts material; working conditions, i.e. frequency of on-off cycles, current and voltage values; device parameters, i.e. the speed of contacts divergence when turned off, the time and amplitude of vibration of the contacts when turned on; environment in which current switching occurs by contacts, etc.

Low-voltage switching devices, especially those types which have mobile contacts of bridge and lobe type should long be in the closed status under different external conditions. It causes strict requirements to stability and level of transition resistance of a contact joint.

There is practically no information in reference books on resistance of contact units of switching devices. Therefore, there is a need for definition of utilization properties of devices.
It is considered that contact resistance consists of two components [8]:

$$
R_{\mathrm{c}}=R_{\mathrm{b}}+R_{\mathrm{t}}
$$

where $R_{\mathrm{b}}$ is the resistance of contacting body ; $R_{\mathrm{t}}$ is the transition resistance of engagement places.

Resistance $R_{\mathrm{t}}$ in contrast to $R_{\mathrm{b}}$ has considerable variations of absolute value and is capable to cause the unpleasant phenomena connected with failure of contacts. The detailed research shows that transition resistance, in turn, consists of two components:

$$
R_{\mathrm{t}}=R_{\mathrm{S}}+R_{\mathrm{c}}
$$

where $R_{\mathrm{S}}$ is the resistance of superficial films; $R_{\mathrm{c}}$ is the tightening resistance.

Earlier it was specified that the superficial films which are formed on contact surfaces can interfere during the current flow. The component of $R_{\mathrm{c}}$ appears due to the fact that the contacts connected end-to-end adjoin not on all visible surface, but only in separate points.

The valid contact points promote current flows in various degrees. Concerning the conductivity, they can be divided into three groups [9]:

- The metal adjoining surfaces;

- The quasimetal adjoining surfaces covered with thickness absorbed by a gas film in several molecules;

- The bearing superficial films with high resistance $R_{\mathrm{s}}$.

Resistance of metal surfaces governs the fact that lines of current are pulled together to places with good conductivity, at the same time current density can reach $10^{7} \mathrm{~A} / \mathrm{cm}^{2}$. Introduction of this concept gave the chance to set quantitative ratios between the contact resistance, physical properties of contact materials, a geometrical form of a surface of engagement and contact effort [10].

The nature of the deformations happening on two adjoining surfaces has a huge impact on contact characteristics. It is usually supposed that the isolating films which are available on a surface collapse only in case of plastic deformation of metal at contact and therefore a large number of researches for the purpose of definition of the factors influencing the mechanism of deformations was conducted. Ways of description of the mechanical contact nature in which mechanical properties of surfaces and their microrelief were considered were developed. Information on detailed structure of surfaces was processed using a computer. This research showed that some parameters of relief, such as radii of microoverhang surface curvature and its distribution by heights, were not always correctly evaluated earlier. For example, it was revealed that for many surfaces roughnesses distribution by height precisely corresponds to the normal distribution law [11].

Thanks to these data, calculation of the loading ratio and the contact area of two surfaces and also definition of the conditions leading to plastic deformation of separate roughnesses became possible. As a result, it 
became clear that irrespective of a deformation type (plastic or elastic), appearing of contact areas is defined not only by the loading attached to two solid bodies, but, generally, by roughness of their adjoining surfaces. This result is in accordance with the general direction which arose recently in physics of the adjoining surfaces. The size of each contact point is $10^{-5}-10^{-4} \mathrm{~cm}$. In terms of a current flow it is clear that engagement happens through the thin isolating barrier to a small amount of far remote spots in it which provide passing of electrons. Chemical, mechanical and thermal nature of microscopic areas of contact on the adjoining surfaces as it was already specified, manages all characteristic of contact.

Further we evaluate the resistance of tightening of contact units of low-voltage devices. Electrical conductance between two contact elements is formed as a result of action of mechanical effort of $P_{\mathrm{c}}$ called contact which presses these elements to each other. One of the major characteristics of the closed electric contacts is the value of the conducting platform between them [12].

The metal tip is formed not on the whole surface, but only in some points. The film which is available on the surface of metal formed by some chemical compounds and having high electrical resistance in some cases can be pressed through under the influence of mechanical efforts, in other cases it can be punched under the influence of electric potential difference. In the place of breakdown, the metal isthmus carrying electric current [13] can be formed.

The main feature of a contact surface is its roughness. Overhangs of the contacting sections adjoin only in some points. Increase in the force of contact clicking leads to growth of a number of such places. Under the influence of electric spark and arc chemical reactions intensively take place as a result of which various chemical compounds having high electrical resistance are formed on the surface of contacts. Besides, corrosion of contacts which in the wet environment has electrolytic character can be observed. The most effective way of fight against films on the contact surface is a mechanical grinding of contacts during inclusion or rather high pressure of one contact on another, capable to press through a film (for gold $P>0.01 \mathrm{H}$, for silver $P>0.1 \mathrm{H}$, for tungsten $P>0.7 \mathrm{H}$ ).

For large films sizes (tens of nanometers) a mechanical wear of surfaces of the contacting metal details can arise, the phenomenon called fritting. If one increases tension at contacts with such films, then transition resistance will quickly decrease.

In case of plastic or elasto-plastic deformation observed in real contacts, the radius of a signal pad $f$ is obtained using the concept of the contact hardness $\mathrm{H}_{\mathrm{v}}$ introduced by R. Holm. Numerically it is equal to the average pressure in contact overhangs which approximately three times exceeds the pressure corresponding to the beginning of plastic deformation [14].

In this case:

$$
f=\left(\frac{P_{\mathrm{c}}}{\pi \xi H_{\mathrm{v}}}\right)^{\frac{1}{2}}
$$

where $\xi$ is the compressibility coefficient considering extent of processing of a contact surface on which its elasto-plastic properties depend; $P_{\mathrm{c}}$ is the contact effort of the electric device; $H_{\mathrm{v}}$ is the contact hardness of material of contact of the switching device.

For purely plastic deformation $\xi=1$, and for elastoplastic $0.3<\xi<1.0$. When the contacts are polished well deformation has almost elastic character, the value $\xi$ can be reduced up to 0.02 . As analytically it is very difficult to display the coefficient $\xi$, we will use its mean value for devices of tens and hundreds of amperes $\xi=0.4$.

For real plane contact the quantity of signal connect pads is $n=3$. Then the radius of a resultant signal connect pad will be defined as

$$
f=\left(\frac{P_{\mathrm{c}}}{n \pi \xi H_{\mathrm{v}}}\right)^{\frac{1}{2}}
$$

Further we give several examples of calculation of radius of a signal connect pad of switching devices. Data for calculation of resistance of tightening are provided by design department of the Divnogorsk Low-Voltage Automatic Circuit-Breakers Works (Divnogorsk, Krasnoyarskiy region).

For AE2040 series automatic machines on rated current 63 A using contact composition is $\mathrm{Ag}(95 \%)$ - C (5\%) for which $H_{\mathrm{v}}=35 \mathrm{~kg} / \mathrm{mm}^{2}, P_{\mathrm{c}}=0.5 \mathrm{~kg}$, the calculated $f=0.67 \mathrm{~mm}$ is used.

For automatic machines of the BA 57-35 series on rated current $250 \mathrm{~A}$ which use $\mathrm{Ag}(85 \%)$ - CdO (15\%) alloy as contact material for which $H_{\mathrm{v}}=55 \mathrm{~kg} / \mathrm{mm}^{2}, P_{\mathrm{c}}=$ $2.5 \mathrm{~kg}$, the calculated $f=0.12 \mathrm{~mm}$ is used.

For automatic machines of the BA 57-39 series on rated current $400 \mathrm{~A}$ which use contact $\mathrm{Ag}(70 \%)-\mathrm{Ni}$ $(30 \%)$ alloy for which $H_{\mathrm{v}}=50 \mathrm{~kg} / \mathrm{mm}^{2}, P_{\mathrm{c}}=4.0 \mathrm{~kg}$, the calculated $f=0.16 \mathrm{~mm}$ is used.

Thus, the received results allow one to evaluate the resistance of tightening of time cut-outs in the general resistance of contact units of devices. So, for example, for the time cut-out of the VA 57-35 brand with $I=250$ $\mathrm{A}$, resistance of a power network is $1.2 \mathrm{mOhm}$, and tightening resistance is $0.12 \mathrm{mOhm}$.

We estimate relationship between power losses of time cut-outs and power which is passed through the device. The power transferred by one pole of the automatic machine is defined according to expression

$$
P=U I \cos \varphi
$$

here $U$ is the mains voltage; $I$ is the current passing through the circuit breaker.

$$
\Delta P=I^{2} k^{2} R
$$


where $k^{2}$ is automatic machine load factor squared; $R$ is resistance of a power network of the switching device (Table 1) [15].

Table 1. Analytical dependence of resistance on rated current.

\begin{tabular}{|l|c|c|}
\hline \multicolumn{1}{|c|}{ Devices } & $\begin{array}{c}\text { Rated } \\
\text { current I, A }\end{array}$ & $\begin{array}{c}\text { Analytical } \\
\text { dependence of } \\
\text { resistance on rated } \\
\text { current }\end{array}$ \\
\hline Magnetic starters & $<70$ & $R=825 / I$ \\
\cline { 2 - 3 } & $\geq 70$ & $R=760 / I$ \\
\hline \multirow{2}{*}{$\begin{array}{l}\text { Time cut-outs and } \\
\text { contactors }\end{array}$} & $<60$ & $R=349 / I$ \\
\cline { 2 - 3 } Safety locks & $\geq 60$ & $R=307 / I$ \\
\cline { 2 - 3 } & $\geq 200$ & $R=210 / I$ \\
\hline \multirow{2}{*}{$\begin{array}{l}\text { Breakers and } \\
\text { package switches }\end{array}$} & Any value & $R=125 / I$ \\
\hline
\end{tabular}

Analytical expressions for determination resilience of switching devices depending on rated current are presented in table 1 .

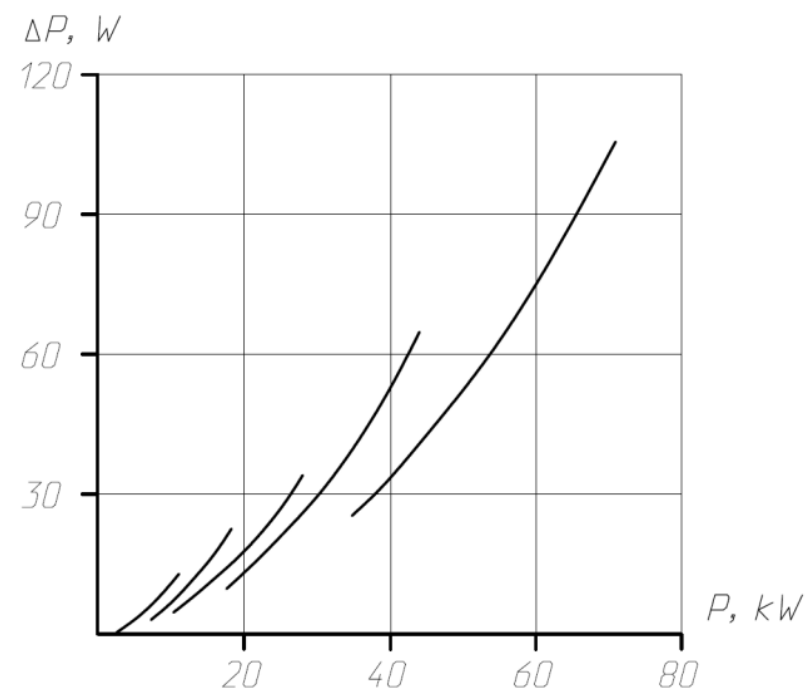

Figure 1. Dependence of power losses on the passed power in time cut-outs.

\section{Conclusion}

The presented characteristics for different groups of time cut-outs dependences of power losses on power (figure 1) which is passed via the switching device are constructed.

Current flow via switching devices causes losses in their pure resistance. The share of these losses is small in comparison with the passed power and is $0.12-0.15 \%$. But while electric power reaches the electric receiver, it passes several switching nodes and a share of losses from delivered power increases up to $1.0-1.5 \%$ that is essential at an electric power loss estimate in shop networks. Therefore, estimating electric power losses in shop networks it is necessary to consider losses in switching equipment.

\section{References}

[1] Yu.K. Rozanov, Electrical and electronic devices (Moscow: Energoatomizdat) 252 (1998)

[2] O.V. Petinov, and E.F. Shcherbakov, Testing of electrical apparatus (Moscow: Higher School) 215 (1985)

[3] V.A. Dulin, Methods for studying the reliability of low-voltage devices (Moscow: Energy) 152 (1970)

[4] K.K. Namitokov, Testing of low voltage apparatus (Moscow: Energoatomizdat) 248 (1985)

[5] M.G. Koblenz, Study of electrical wear resistance power contacts of contactors when operating in intermittent mode (Electrical contacts) (Moscow: Gosenergoizdat) 59-71 (1962)

[6] E.A. Konyukhova, Reliability of power supply to industrial enterprises (Moscow: NTF Energoprogress) 92 (2001)

[7] V.V. Shevchenko, and E.I. Gracheva, Determination of electric power losses in shop networks with voltage up to $1000 \mathrm{~V}$ Industrial power engineering. 10, 33-35 (2001)

[8] Yu.S. Zhelezko, A.V. Artemyev, and O.V. Savchenko, Calculation, analysis and rationing of electricity losses in electric networks (Moscow: ENAS Center) (2003)

[9] O.V. Fedorov, Assessment of influence parameters of electric equipment at a size of losses the electric power in intra factory networks of low voltage 2nd Int. Conf. on Industrial Engineering, Applications and Manufacturing. CFP16F42-ART. DOI: 10.1109/ICIEAM.2016.7911463 (2016)

[10] G.V. Butkevich, N.A. Belkin, and N.A. Vedeshenkov, Electric erosion of high-current contacts and electrodes (Moscow: Energy) 256 (1978)

[11] A.N. Shpiganovich, A.A. Shpiganovich, and E.P. Zatsepin, Features of the functioning multi-level power supply systems.News from higher educational institutions of the Black Earth Region. 3(53), 12-24 (2018)

[12] A.N. Shpiganovich, and A.A. Shpiganovich, To assess the reliability parameters of the equipment for power supply systems. News of the higher educational institutions of the Black Earth Region. 4(46), 48-56 (2016)

[13] E.I. Gracheva, and N.A. Kopytova, Analysis structure of systems shop power supply of the enterprises the machine-building industry. News of higher educational institutions. Power problems. 5(6), 73-78 (2011)

[14] R. Holm, Electric contacts (Moscow: Energy) 456 (1978)

[15] E.I. Gracheva, A.R. Safin, and A.V. Shagidullin, Modeling of the influence changes in electrical equipment parameters on the value of the equivalent resistance the intrashop power supply systems. Elektrika. 7, 2-6 (2013) 